\title{
Spectrum Estimation by Wavelength Shift Time-Stamping in a Fiber Bragg Grating Sensor
}

\author{
Kalaga Venu Madhav, Student Member, IEEE, and S. Asokan
}

\begin{abstract}
We propose and numerically demonstrate a novel technique for estimating the spectral content of the perturbation on a Fiber Bragg grating (FBG) sensor. The system utilizes an FBG array as a wavelength reference for time-stamping the wavelength shift of the sensor. This technique has demonstrated a good agreement between the actual and estimated spectrum. In addition to having the advantage of selecting the operational window of the sensor, it also gives an absolute measurement of the perturbation.
\end{abstract}

Index Terms-Fiber Bragg grating (FBG), optical sensor, spectrum, wavelength detection.

\section{INTRODUCTION}

$\mathbf{T}$ HE SMALL dimensions of a fiber Bragg grating (FBG) sensor provide unique capabilities for a nonobtrusive method of sensing various parameters in aerospace, civilian, military, and industrial scenarios. Of particular importance is its usefulness as an embedded sensor in composite structures. The self-referencing feature of the FBG makes it immune to intensity changes and electromagnetic noise. Various methods such as ratiometric approach, wavelength-division multiplexer, biconical fiber filters, Fabry-Pérot filters, acoustooptic filters, Bragg grating-based filters, Mach-Zehnder interferometer, wavelength-tunable fiber lasers, charged-coupled device spectrometers, and Fourier transform spectroscopy have been demonstrated [1]. A minimum variance shift technique [2] and a polarization-maintaining fiber loop mirror [3] have also been demonstrated for wavelength demodulation of an FBG sensor. A minimum intensity based detection for absolute strain measurement is described in [4]. Recently, an arrayed waveguide grating as wavelength interrogator has been demonstrated [5].

In some applications, it is more desirable to know the frequencies involved in the perturbation on an FBG. Apart from absolute measurements of strain, load, temperature, vibration, or acceleration, a direct knowledge of their spectral content is highly advantageous for studying the structural stability and integrity at extreme conditions. FBG sensors used for analysis of perturbation events occuring in environments similar to ballistic testing and shock waves arising due to structural fracture under extreme conditions, require very high speed wavelength demodulation methods. Such events not only contain high frequencies but also occur in a very small span of time. Demodulation schemes employing active elements such as scanning Fabry-Pérot interfer-

Manuscript received December 17, 2003.

The authors are with the Department of Instrumentation Indian Institute of Science, Bangalore 560 012, India (e-mail: kalaga@isu.iisc.ernet.in; sasokan@ isu.iisc.ernet.in).

Digital Object Identifier 10.1109/LPT.2004.826080

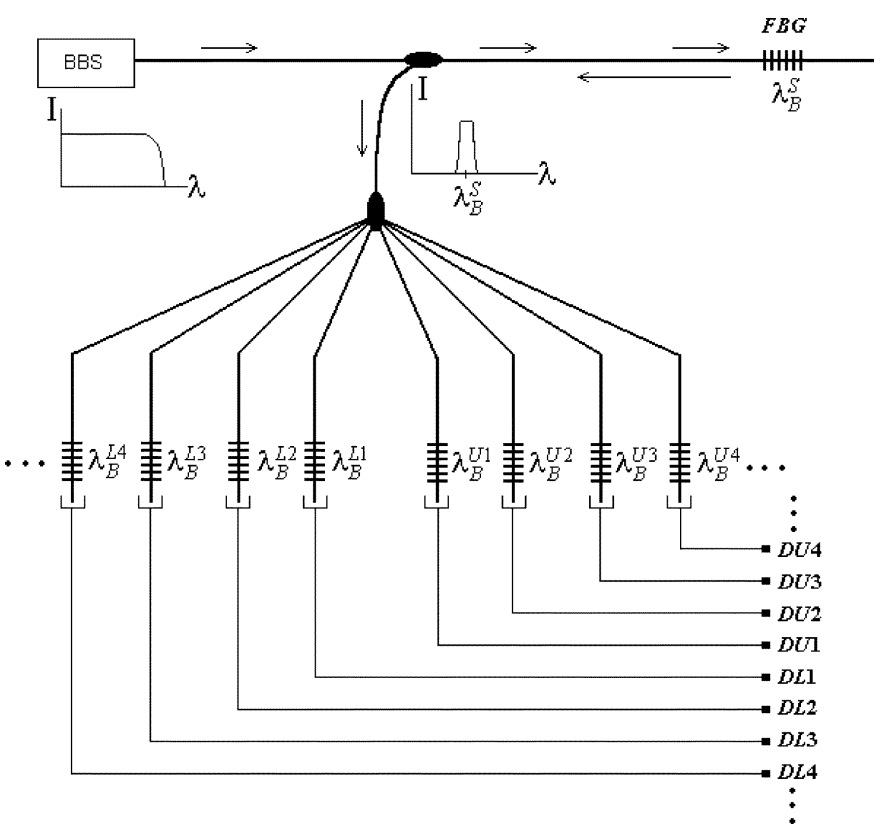

Fig. 1. Schematic diagram of the WSTS architecture for the FBG sensor.

ometer cannot operate at speeds higher than a few kilohertz. At higher speeds, a self-referencing passive demodulation scheme requiring no scanning is an ideal choice.

In this letter, we describe a wavelength shift time-stamping (WSTS) method for estimating the spectrum of perturbation. An FBG array as a wavelength reference creates the time stamps at instances where the sensor wavelength matches the wavelengths of the reference FBGs. Theory and simulated evaluations of this technique are described in the following sections.

\section{THEORY}

The proposed layout of the WSTS technique is shown in Fig. 1. Here, the wavelengths of the reference arm FBGs $\lambda_{B}^{\mathrm{Ln}}$ and $\lambda_{B}^{\mathrm{Un}}$ are chosen to satisfy the following relations:

$$
\begin{aligned}
\lambda_{B}^{\mathrm{Ln}} & =\lambda_{B}^{S}-n \delta \lambda \\
\lambda_{B}^{\mathrm{Un}} & =\lambda_{B}^{S}+n \delta \lambda \\
\delta \lambda & >\frac{1}{2}\left\{[\mathrm{FWHM}]_{B}^{S}+[\mathrm{FWHM}]_{B}^{\mathrm{Ln}, \mathrm{Un}}\right\} \\
{[\mathrm{FWHM}]_{B}^{S} } & \leq[\mathrm{FWHM}]_{B}^{\mathrm{Ln}, \mathrm{Un}}
\end{aligned}
$$

where $n=1,2,3, \ldots, k$, and $\delta \lambda$ is the separation between the wavelengths. Condition (3) ensures that there is no overlap of the wavelengths in the absence of any disturbance. The strain and temperature sensitivity for a germania-doped silicon fiber 


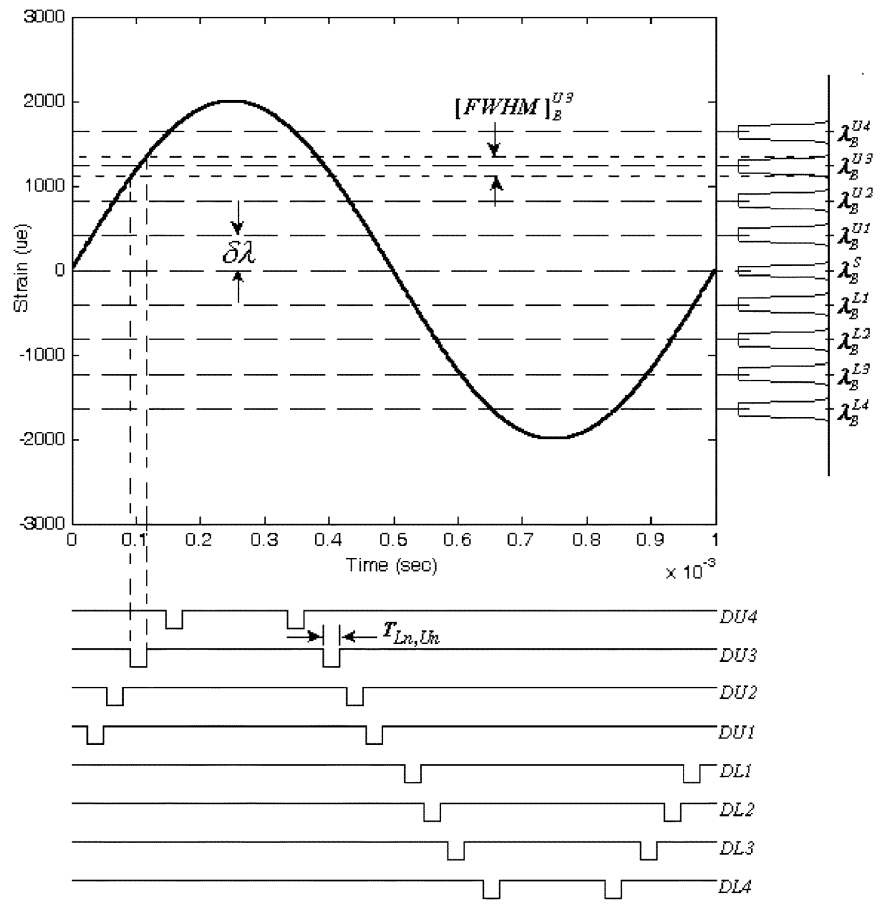

Fig. 2. Time stamps produced by a sinusoidal strain of $1-\mathrm{kHz}$ frequency.

$\left(n_{\mathrm{eff}}=1.482\right)$ are $1.22 \mathrm{pm} / \mu \varepsilon$ and $13.7 \mathrm{pm} /{ }^{\circ} \mathrm{C}$, respectively, for $\lambda_{B}^{S} \approx 1560 \mathrm{~nm}$, where $\lambda_{B}^{S}=2 n_{\text {eff }} \Lambda$.

Keeping this calibration as the reference, the relation

$$
\Delta \lambda_{B}^{S} \geq n \delta \lambda
$$

is satisfied for the WSTS technique, where $\Delta \lambda_{B}^{S}$ is the change in the sensor wavelength due to perturbation. The condition for $n=1$ decides the resolution of measurement of strain and temperature through the relations [6]

$$
\begin{aligned}
\varepsilon_{z} & =\frac{n \delta \lambda}{\lambda_{B}^{S}\left(1-p_{e}\right)} \\
\delta T & =\frac{n \delta \lambda}{\lambda_{B}^{S}\left(\alpha_{\Lambda}+\alpha_{n}\right)}
\end{aligned}
$$

where $p_{e}$ is the effective stress-optic coefficient $(\sim 0.2126), \alpha_{\Lambda}$ is the thermal expansion coefficient $\left(\sim 0.55 \times 10^{-6}\right)$, and $\alpha_{n}$ is the thermooptic coefficient $\left(\sim 8.6 \times 10^{-6}\right)$, for the optical fiber. Substituting these constants in (6) and (7) for $\delta \lambda=0.1 \mathrm{~nm}$, the minimum strain and temperature change that can be measured by the WSTS technique are $\sim 81.5 \mu \varepsilon$ and $\sim 7^{\circ} \mathrm{C}$, respectively. For $n=1$, if the condition in (4) and the equality in (5) is satisfied, then $\lambda_{B}^{\mathrm{L} 1}=\lambda_{B}^{S}-\Delta \lambda_{B}^{S}$ for a compressive strain (or lower temperature), or $\lambda_{B}^{\mathrm{U1}}=\lambda_{B}^{S}+\Delta \lambda_{B}^{S}$ for a tensile strain (or higher temperature). Consequently, the output of the photodetector DL1 or DU1 will go low, as a result of wavelength match. Similarly for $n=2$, the outputs of photodetector DL2 or DU2 will go low when there is a match between $\lambda_{B}^{S} \mp \Delta \lambda_{B}^{S}$ and $\lambda_{B}^{\mathrm{L} 2}$ or $\lambda_{B}^{\mathrm{U} 2}$, respectively. The output of the corresponding photodetector will go low when the perturbed $\lambda_{B}^{S}$ matches $\lambda_{B}^{\mathrm{Ln} \text {, Un }}$, creating a time stamp. Fig. 2 shows the time stamps produced by a sinusoidal strain perturbation of frequency $1 \mathrm{kHz}$ on the sensor FBG. In other words, the strain is sampled at the time instances

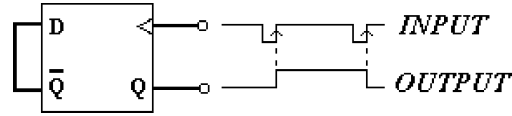

Fig. 3. DFF circuit.

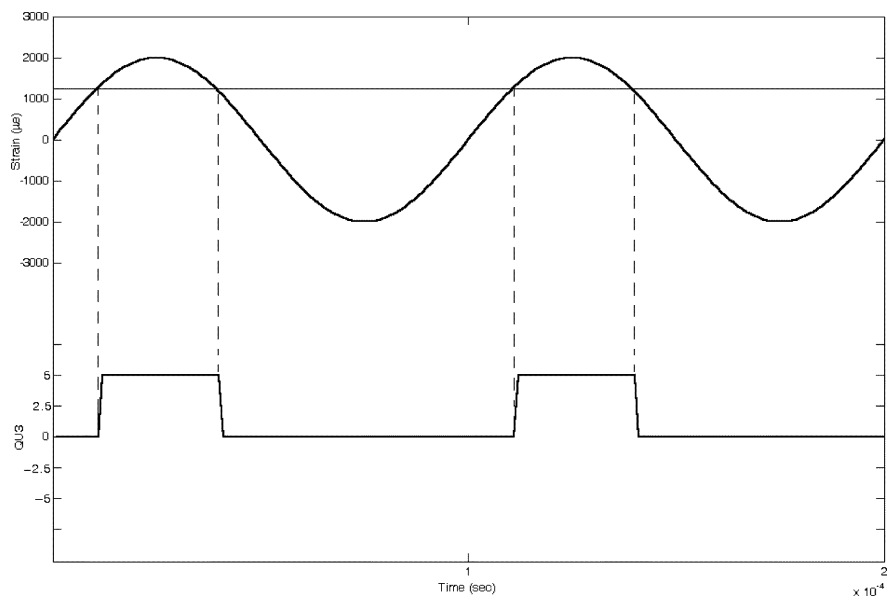

Fig. 4. Output $Q U 3$ for a sinusoidal tensile strain exceeding $3 \delta \lambda$.

where the wavelength $\lambda_{B}^{S}$ matches $\lambda_{B}^{\mathrm{Un}}$ or $\lambda_{B}^{\mathrm{Ln}}$. The pulsewidth $T$ of the time stamps is given as

$$
T_{\mathrm{Ln}, \mathrm{Un}}=[\mathrm{FWHM}]_{B}^{\mathrm{Ln}, \mathrm{Un}} .
$$

\section{Simulation}

The WSTS technique is numerically evaluated for $n=4$, $\delta \lambda=0.5 \mathrm{~nm}$, and $\lambda_{B}^{S}=1560 \mathrm{~nm}$. For this choice of $n$ and $\delta \lambda$, the maximum and minimum strain that can be absolutely measured are \pm 1639.344 and $\pm 409.836 \mu \varepsilon$, respectively. Before the spectral contents of the photodetector outputs (DUn, DLn) are estimated, they are subjected to preprocessing using a D-flip flop (DFF) to generate signals (QUn, QLn). The output from each of the photodetectors (DUn, DLn) is conditioned to comply with transistor-transistor logic (TTL) levels and is given as the clock input of the DFF, where the $\bar{Q}$ output of the $\mathrm{DFF}$ is feedback to the input $\mathrm{D}$, as shown in Fig. 3. This simple circuit creates a digital signal (QUn, QLn) that is at TTL logic-1 as long as the perturbation satisfies condition (5), for a given $n$. For example, QU3 will be at logic-1 as long as $\Delta \lambda_{B}^{S} \geq 3 \delta \lambda$, for a tensile sinusoidal strain, as shown in Fig. 4. It is evident from Fig. 4 that the fundamental frequency of the signal QU3 is same as that of the sinusoidal strain perturbation. Hence, a direct power spectral density (PSD) of QU3 reveals the spectral content of the perturbation on the sensor FBG. To test the PSD reproducibility of the WSTS technique, an arbitrary strain perturbation, as shown in Fig. 5, is applied to the FBG sensor. For the choice of $n$ and $\delta \lambda$ mentioned above, time stamps are created corresponding to the sample points, where the strain perturbation crosses $\pm 409.836, \pm 819.672, \pm 1229.508$, and $\pm 1639.344 \mu \varepsilon$. Subjecting each of the photodetector outputs to preprocessing using DFFs, the PSDs of the signals QUn and QLn are plotted in comparison with the actual PSD of the perturbation in Figs. 6 and 7, after applying the Kaiser window, with $N=50$ and $\beta=1$. 


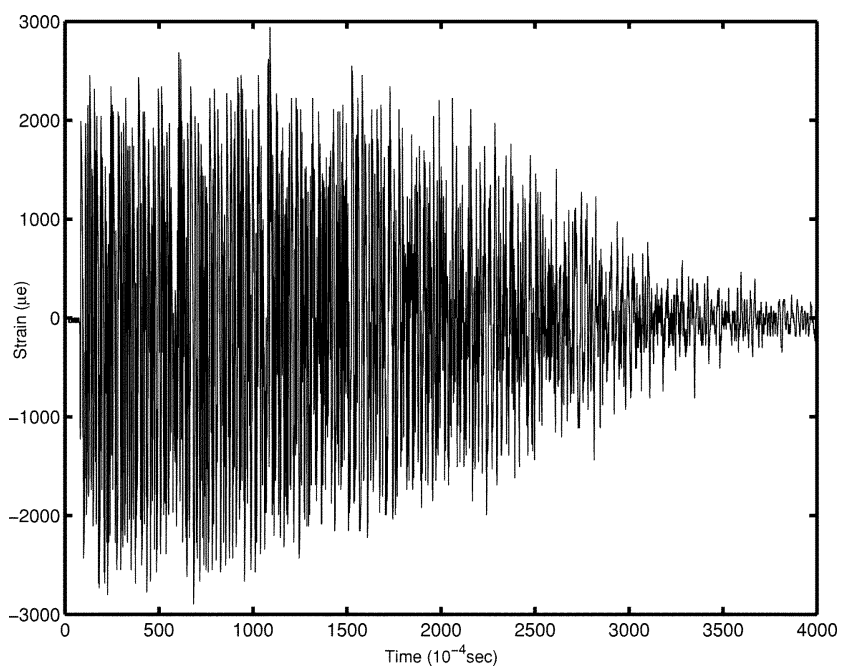

Fig. 5. Arbitrary strain perturbation applied on the sensor FBG.

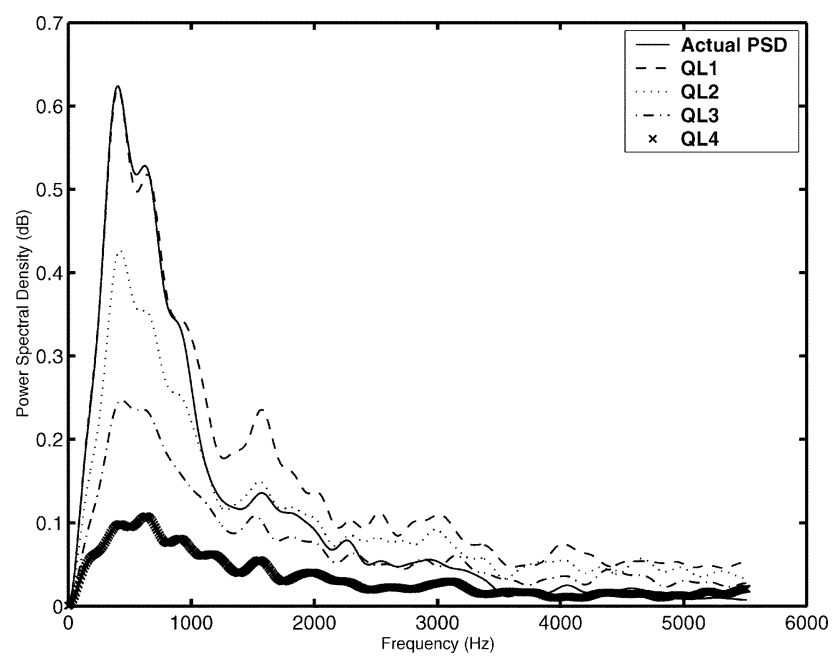

Fig. 6. PSD of the signals QL1, QL2, QL3, and QL4 compared with the actual PSD of the strain.

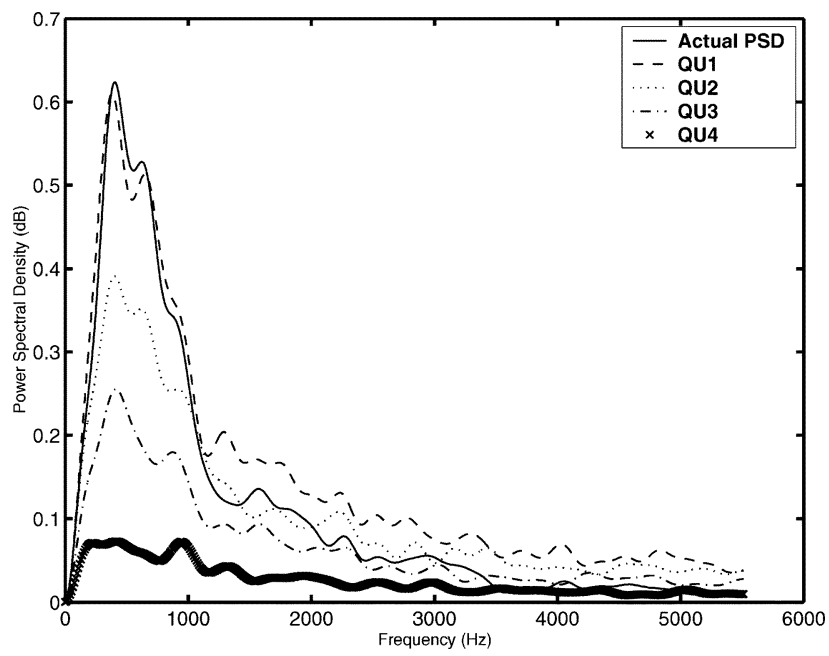

Fig. 7. PSD of the signals QU1, QU2, QU3, and QL4 compared with the actual PSD of the strain.

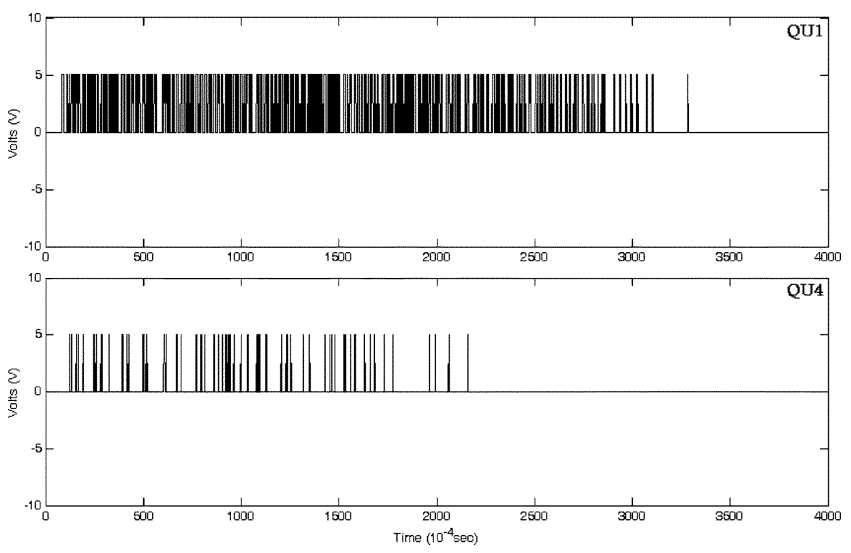

Fig. 8. Signals QU1 and QU4.

\section{RESULTS AND DISCUSSION}

It is observed that the PSDs of the signals QUn and QLn agree with the true PSD of the perturbation. It is also observed that the PSDs of QU1 and QL1 show the most agreeable spectral profile match, when compared to the PSDs of QU4 and QL4. This observation can be explained by comparing the total "information content," for example, in QU1 and QU4, by referring to Fig. 8. Evidently, the perturbation on the FBG sensor is such that there are more instances of wavelength match between $\lambda_{B}^{S}$ and $\lambda_{B}^{\mathrm{U} 1}$, than between $\lambda_{B}^{S}$ and $\lambda_{B}^{U 4}$. Knowing $n, \delta \lambda, \lambda_{B}^{S}$, and the temporal location of the time stamps (sample points) at the output of the photodetectors, the perturbation signal can be reconstructed by assembling the sample points and the amplitudes derived from $n \delta \lambda$.

\section{CONCLUSION}

A novel technique for estimating the spectral content of the perturbation on an FBG sensor has been proposed and its performance is numerically evaluated. The technique is based on collecting the samples of the perturbation across an FBG array used as filters. The samples are time stamped at the instances of wavelength match, and preprocessed before their spectral densities are extracted. Very good agreement between the actual PSD and the derived PSDs, is demonstrated. The proposed WSTS technique is completely a passive interrogation system for wavelength demodulation and spectrum estimation, ideal for very high speed applications.

\section{REFERENCES}

[1] A. D. Kersey, M. A. Davis, H. J. Patrick, M. LeBlanc, K. P. Koo, C. G. Askins, M. A. Putnam, and E. J. Friebele, "Fiber grating sensors," J. Lightwave Technol., vol. 15, pp. 1442-1463, Aug. 1997.

[2] J. M. Gong, J. M. K. MacAlpine, C. C. Chan, W. Jin, M. Zhang, and Y. B. Liao, "A novel wavelength detection technique for fiber Bragg grating sensors," IEEE Photon. Technol. Lett., vol. 14, pp. 678-680, May 2002.

[3] S. Chung, J. Kim, B.-A. Yu, and B. Lee, "A fiber Bragg grating sensor demodulation technique using a polarization maintaining fiber loop mirror," IEEE Photon. Technol. Lett., vol. 13, pp. 1343-1345, Dec. 2001.

[4] B. H. Lee, "Absolute Strain Measurement using Fiber Bragg Grating," Ph.D. dissertation, Univ. of Colorado, Boulder, 1996.

[5] Y. Sano and T. Yoshino, "Fast optical wavelength interrogator employing arrayed waveguide grating for distributed fiber Bragg graing sensors," J. Lightwave Technol., vol. 21, pp. 132-139, Jan. 2003.

[6] G. Mertz and W. W. Morey, "Bragg grating formation and germanosilicate fiber photosensitivity," Proc. SPIE, vol. 1516, pp. 185-199, 1991. 